\title{
Ein ungarisches Handbuch über rhetorische und stilistische Figuren
}

István Szathmári et al. (Hg.), turwissenschaftler sowie Lehrer von Alakzatlexikon. A retorikai és Lehranstalten der Sekundarstufe, stilisztikai alakzatok kézikönyve [Figurenlexikon. Handbuch der rhetorischen und stilistischen Figuren]. Budapest: Tinta Könyvkiadó, 2008. 595 S.

Das vorliegende Werk ist das erste Figurenlexikon in ungarischer Sprache. Es wurde unter Leitung von Professor István Szathmári von einer 29-köpfigen Stilforschungsgruppe (Stíluskutató Csoport) am Institut für Ungarische Sprachwissenschaft der Eötvös-Lorand-Universität erstellt. Bei den Mitgliedern der Gruppe handelt es sich in der Regel um Lehrkräfte verschiedener Universitäten, Sprach- oder Literadie vorwiegend im Bereich der Stilistik tätig sind. Das Werk hat kaum ungarischsprachige Vorgänger, weshalb die Verfasser sich bei der Zusammenstellung der Stichwortartikel lediglich auf das Világirodalmi Lexikon [Lexikon der Weltliteratur] und auf das Werk Kis magyar retorika [Kurze ungarische Rhetorik] von Zoltán G. Szabó und László Szörényi (Budapest 1988) stützen konnten, die freilich nur für einige Artikel die Grundlage lieferten.

Angestrebt wurde ein modernes, auf die heutigen Bedürfnisse zugeschnittenes, strikt wissenschaftlich begründetes, aber auch die praktische Arbeit (z. B. schulischen und 
anderen Unterricht) unterstützendes Nachschlagwerk.

Die Figuren werden in der Reihenfolge der Stichwortartikel behandelt. Die Anzahl der eigentlichen Artikel beträgt 196, aber zusätzlich werden 129 Stichwörter angeführt, mit deren Hilfe der Leser weitere Informationen auffinden kann.

Bis zur Abfassung des Buches war der Begriff Figur nicht einmal exakt definiert. Die Einleitung bietet die folgende Definition: „Eine Figur ist eine konventionalisierte und tradierte sprachliche Form (Wortform, Redewendung oder Ausdrucksweise), deren Verwendung die Sprache stilistisch oder rhetorisch wirkungsvoller macht. Folglich ist eine Figur gleichzeitig Struktur (Form) und Verwendungsweise der Sprache (Funktion). Eine Figur ist als kodifiziertes sprachliches Sem hinsichtlich ihres Ursprungs das Resultat einer modifizierenden Handlung, in deren Verlauf der Sprecher aus kommunikativen Gründen von der gewohnten, als normativ geltenden sprachlichen Form abweicht; diese neue Struktur bildet sich im Denken des Sprechers und kategorisiert sich als rhetorische Erscheinung." (7).

Der Chefredakteur zeigt ferner, dass sich mit der Interpretation der Figuren weitere wichtige Fragen verbinden. Eine dieser Fragen ist die sog. Offenheit, denn als Ergebnis einer vielfältigen und wechselhaften
Kommunikation können immer neue Figuren entstehen. Typisch ist ferner, dass der kognitiven Auffassung entsprechend die betreffende sprachliche Form gleichzeitig auch funktional ist. Figuren sollten nicht allein als Zierwerk der Sprache betrachtet werden, denn sie dienen ihr als wesentliche Elemente des vollkommenen Ausdrucks und der Verständlichkeit. Wesentlich ist zudem, dass die Figuren neben Effektmitteln auch wichtige Stilmittel sind. Natürlich verbinden sich mit dem Figurensystem und dem Wesen der Figuren auch Kontroversen. Zu diesen zählt nach Ansicht des Chefredakteurs die Abweichung, d. h. eine Figur, die auf einen Fehler zurückgeht. Im vorliegenden Werk wird die Verwendung des Ausdrucks auf einen Fehler zurückgehend vermieden, stattdessen sprechen die Verfasser recht systematisch von Abweichung.

Der Chefredakteur geht auch auf die Frage ein, ob der Tropus zu den Figuren zählt. Dabei kristallisiert sich der Gedanke heraus, dass die Sprachbilder in der Hauptsache kognitive Abläufe, die Figuren dagegen sprachlich-syntaktische Vorgänge voraussetzen. Der Tropus wurde also einbezogen, wird aber nicht aus stilistischer Sicht betrachtet, sondern in einem separaten Stichwortartikel über die Problematik der Tropen im Verhältnis zu den Figuren behandelt. 
Die erwähnten Fragen gehören jedoch nicht zu den umstrittensten. Die größten Kontroversen betreffen die Einordnung der Figuren aus sprachlich-grammatischer Sicht (kann man, und wenn ja, unter welchen Umständen von Laut-, Wort-, Satz- oder Gedankenmustern sprechen). Die Verfasser sind zu dem Ergebnis gekommen, dass man die Figuren bei Bedarf der sprachlichen Ebene entsprechend klassifizieren kann, halten es jedoch für wichtig, sie nicht taxonomisch zu klassifizieren. Stattdessen halten sie sich bei der Klassifizierung an semantische und pragmatische Gesichtspunkte und verweisen auf die grammatische Struktur der Figur.

Es ist wichtig, dass das Werk relativ viele Stichwortartikel enthält. So können einzelne Figuren und Figurentypen eingehend behandelt werden. Alle Artikel sind meiner Einschätzung nach das Ergebnis gründlicher Arbeit. Auch bei der Wahl der Stichwörter wurde ein möglichst umfassendes Spektrum angestrebt. Am Anfang jedes Artikels stehen das Stichwort selbst und unmittelbar danach - zur Vermeidung von Missverständnissen und mit Rücksicht auf fremdsprachige Leser - die Bezeichnungen der Figuren in den wichtigsten Fremdsprachen: Griechisch, Latein, Deutsch, Französisch, Englisch und Russisch. Danach folgt eine kurze Definition der Figur, die dem Leser unmittelbar eine Vorstellung vom Inhalt des Artikels vermittelt. Anschließend geht es um den geschichtlichen Hintergrund der Figur, wobei natürlich auch die Personen, mit denen die betreffende Figur verbunden ist, und die Verwendung des Terminus $\mathrm{zu}$ verschiedenen Zeiten erwähnt werden. Zum Schluss wird die heutige Bedeutung des Stichworts angegeben. Ich betrachte es als besonders gute Lösung, dass jedem Stichwortartikel prototypische Beispiele beigefügt sind, die das jeweilige Phänomen veranschaulichen. Die Beispiele enthalten in lobenswertem Umfang Material aus verschiedenen Bereichen, von der Belletristik über die Publizistik bis hin zur Alltagsund Umgangssprache und sogar bis zum Slang. Diese Vorgehensweise kommt den Bedürfnissen ungarischer und möglicherweise auch fremdsprachiger Leser entgegen und verhindert Missverständnisse.

Es sei erwähnt, dass jeder Stichwortartikel einen einzigen Verfasser hat, dessen Initialen unter dem Artikel stehen. Den eigentlichen Textteil der Stichwortartikel beschließt ein Quellenverzeichnis, das neben ungarischen Quellen auch ausländische (internationale) Literatur enthält. Die beträchtliche Anzahl an Referenzwörtern ist notwendig, denn sie zeigen an, unter welchem Stichwort sich Informa- 
tionen zu dem im jeweiligen Artikel behandelten Begriff finden.

Die Stichwortartikel sind natürlich kurz, aber sowohl aus theoretischer wie aus praktischer Perspektive tiefschürfende, gründliche Darstellungen von hohem Nutzwert. Die theoretischen Erläuterungen vermitteln einen guten Eindruck von der Forschung zu dem jeweiligen Stichwort. Gleichzeitig geben sie Aufschluss über die eigenen Forschungsergebnisse und Auffassungen des jeweiligen Verfassers. Man kann feststellen, dass die Autoren der einzelnen Artikel die eminentesten ungarischen Experten für die jeweilige Figur sind und sie häufig auch erforscht haben. Der Chefredakteur und die Verfasser haben gemeinsam über jedes Stichwort diskutiert und so den betreffenden Artikel, seinen Aufbau, die darin geäußerten Positionen und die Struktur ergänzt und kritisch beurteilt, wobei jedoch stets die Meinung des Verfassers im Vordergrund stand. Das Lexikon ist somit das Ergebnis sowohl individueller als auch kollektiver Arbeit, da die Gemeinschaft der Verfasser beim Entstehen des Werks zu einem gewissen Grad eine vereinheitlichende und ergänzende Rolle spielte.

$\mathrm{Da}$ es in diesem Rahmen unmöglich wäre, die Stichwörter im Einzelnen vorzustellen und zu beurteilen, erwähne ich nur einige, um zu zeigen, welche Arten von Stichwörtern (Begriffen) gewählt wurden, z. B. ung. abúzió oder katakrézis; ung. epentézis; ung. metafora usw. Weitere Stichwörter dieses Typs sind adjekció, adjunkció, aferézis, akumen, alakzat (Figur), alakzat és norma (Figur und Norm), alakzat és poétika (Figur und Poetik), alakzat és pragmatika (Figur und Pragmatik), alakzat és retorika (Figur und Rhetorik) usw.

Die Stichwortartikel weichen natürlich im Umfang voneinander $a b$, sind jedoch in Ausdruck und Aufbau klar und trotz ihrer relativen Kürze inhaltsreich.

Es ist hervorzuheben, dass in den Stichwortartikeln häufig neben der Definition des eigentlichen Stichworts, d. h. des jeweiligen Begriffs auch wissenschaftliche Fragen behandelt werden, die eng mit dem Begriff verbunden und bei der Betrachtung notwendigerweise zu erörtern sind (z. B. finden sich unter dem Stichwort Figur nachgeordnete Stichwörter folgender Art: Figur und Grammatik: 1. Die Figuren als sprachliche Gebilde, 2. Beurteilung und Gliederung der Figuren, 3. Sprachfehler und rhetorische Tugend; Figur und Norm, Figur und Poetik, Figur und Pragmatik, Pragmatische Aspekte in der Tradition der Rhetorik, Pragmatische Aspekte in der sprachlich-funktionalen Beschreibung der Figuren, Die Proble- 
matik der Figuren in der Pragmatik, Figur und Rhetorik: 1. Die Position der Figuren im System der Rhetorik, 2. Die Betrachtung der Figuren in der Geschichte der Rhetorik, 3. Die Interpretation der Figuren im Kategoriensystem der Rhetorik, rhetorische Tugend, stilistische Zierde - sprachlicher Verstoß, Regelmäßigkeit - Abweichung, 4. Figurentypen und Klassifikation der Figuren: Beschreibung und Frequenz, 5. Das Verhältnis zwischen Tropen und Figuren in der Rhetorik, sowie 6. Zusammenfassung. Darauf folgen die rhetorische Tradition der Figurenforschung und danach der Teil Figur und Stilistik, der wie folgt gegliedert ist: 1 . Klassische Rhetorik und Stilistik, 2. Die Differenzierung der Stilistik als eingenständiger Teilbereich, die Entstehung der funktionalen Stilistik, 3. Die Ausweitung der theoretischen Grundlagen der Stilistik, 4. Kognitive Stilistik, 5. Zusammenfassung (23-54). Des Weiteren werden Fragen behandelt, die Figur und Semantik (54-) sowie Figurenfunktionen und Verbindungen von Figuren (56-62) betreffen.

Mit den aufgezählten Themen verbinden sich gründliche Überlegungen, die in enger Verbindung zur Thematik und Gedankenwelt der heutigen sprachwissenschaftlichen Forschung stehen. Der Abschnitt über die Metapher (390-402) ist trotz seiner Kürze ein vorbildlich verfasster Stichwortartikel, in dem u. a. die Funktionen der Metapher, ihre sprachliche und ästhetische Rolle, ihre Nuancen und ihr anpassendes Wesen sowie Besonderheiten der kognitiven Metaphernlehre erläutert werden. Unter dem Stichwort Metonymie ist $\mathrm{zu}$ lesen, dass die Themenkreise, mit denen man die der Metonymie zugrundeliegenden verwandten Begriffe zu präzisieren sucht, in der einschlägigen Literatur auf vielerlei Weise klassifiziert werden (die Beziehung zwischen der Zeit und den in dieser Zeit Lebenden, die Beziehung zwischen dem Ort und den dort Lebenden, die Beziehung zwischen Ursache und Wirkung usw.) (410-420).

Ich halte das vorliegende Werk aufgrund seiner Thematik, seines anspruchsvollen theoretischen Charakters und seiner modernen Anschauungsweise für überaus wertvoll und interessant. Es ist aufschlussreich für Forscher des Fachs und an Stilistik Interessierte, aber auch für Lernende im Universitätsund Sekundarschulbereich von Nutzen. Seinem Inhalt nach ist das Werk enzyklopädisch, die wesentlichen Züge der Figuren werden in den Stichwortartikeln übersichtlich dargestellt. Meiner Ansicht nach wird es bedeutenden Einfluss auf Leser haben, die sich für Literatur oder Sprachwissenschaft interessieren. Wohl aus diesem Grund 
Ein hervorragendes Werk über die Geschichte der Forschung...

veröffentlichten der Chefredakteur, unter anderen Interessierten die BeProfessor István Szathmári, und schäftigung mit Stilistik und Rhetoder Verlag Ende 2010 eine verein- rik fördert.“ Dieses kleine Lexikon fachte, gekürzte und popularisierte bleibt aus verständlichen Gründen Fassung des Werks (Stíluseszközök hinsichtlich Arbeitsaufwand, Umés alakzatok kislexikona [Kleines fang und theoretischer Tiefe weit Lexikon der Stilmittel und Figu- hinter dem oben vorgestellten Werk ren], Budapest: Tinta Könyvkiadó, zurück, wird aber dank seiner knap142 S.). Der Verfasser schreibt: „Ich peren Form den Bedürfnissen eines hoffe, dass auch dieses kleine Lexi- breiteren Publikums gerecht. kon seine Aufgabe erfüllt und vor allem unter Studenten, aber auch

István Nyirkos 